\title{
Impact of preoperative fecal short chain fatty acids on postoperative infectious complications in esophageal cancer patients
}

Masaaki Motoori ${ }^{1,2 *}$, Koji Tanaka $^{1,3}$, Keijiro Sugimura ${ }^{1}$, Hiroshi Miyata ${ }^{1}$, Takuro Saito ${ }^{1,3}$, Yasuhiro Miyazaki², Kazumasa Fujitani ${ }^{2}$, Yukiko Kado ${ }^{4}$, Takashi Asahara ${ }^{4}$ and Masahiko Yano ${ }^{1}$

\begin{abstract}
Background: The intestinal epithelial barrier allows absorption of dietary nutrients and prevents passage of pathogens and toxins into the body. Severe insults have a negative impact on the intestinal environment, which may decrease intestinal barrier function and cause bacterial translocation. Bacterial translocation, which can cause infectious complications, is defined as the passage of microbes from the gastrointestinal tract across the mucosal barrier to extraintestinal sites. The aim of this study was to investigate the correlation between concentrations of preoperative fecal organic acids and the occurrence of postoperative infectious complications in patients with esophageal cancer.
\end{abstract}

Methods: Fifty-five patients with esophageal cancer who underwent esophagectomy were enrolled in this study. Perioperative synbiotics were administered to all patients. Perioperative clinical characteristics and concentrations of preoperative fecal organic acids were compared between patients with and without postoperative infectious complications.

Results: Postoperative infectious complications occurred in 10 patients. In patients with complications, the concentrations of acetic acid and propionic acid were significantly lower than in patients without complications ( $p=0.044$ and 0.032 , respectively). The concentration of butyric acid was nonsignificantly lower in patients with complications, while the concentration of lactic acid was nonsignificantly higher. The calculated gap between the concentrations of fecal acetic acid plus propionic acid plus butyric acid minus lactic acid was significantly lower in patients with complications. Multivariate analysis revealed that a low gap between acetic acid plus propionic acid plus butyric acid minus lactic acid was an independent risk factor for postoperative infectious complications ( $p=0.027)$.

* Correspondence: mmotoori@gh.opho.jp

'Department of Surgery, Osaka International Cancer Institute, 3-1-69 Otemae, Osaka, Chuo-ku 541-8567, Japan

${ }^{2}$ Department of Surgery, Osaka General Medical Center, 3-1-56 Bandai-higashi, Osaka 558-8558, Japan

Full list of author information is available at the end of the article

C C The Author(s). 2020 Open Access This article is licensed under a Creative Commons Attribution 4.0 International License, which permits use, sharing, adaptation, distribution and reproduction in any medium or format, as long as you give appropriate credit to the original author(s) and the source, provide a link to the Creative Commons licence, and indicate if changes were made. The images or other third party material in this article are included in the article's Creative Commons licence, unless indicated otherwise in a credit line to the material. If material is not included in the article's Creative Commons licence and your intended use is not permitted by statutory regulation or exceeds the permitted use, you will need to obtain permission directly from the copyright holder. To view a copy of this licence, visit http://creativecommons.org/licenses/by/4.0/. The Creative Commons Public Domain Dedication waiver (http://creativecommons.org/publicdomain/zero/1.0/) applies to the data made available in this article, unless otherwise stated in a credit line to the data. 
(Continued from previous page)

Conclusions: Preoperative fecal concentrations of organic acids had a clinically important impact on the occurrence of postoperative infectious complications in patients with esophageal cancer. To reduce postoperative infectious complications, it may be useful to modulate the intestinal environment and maintain concentrations of fecal organic acids before surgery.

Keywords: Esophageal cancer, Esophagectomy, Postoperative complications, Intestinal environment, Organic acids

\section{Background}

Subtotal esophagectomy for esophageal cancer is one of the most invasive gastrointestinal surgeries and is associated with high morbidity and mortality rates, though there have been recent advances in surgical techniques and perioperative management $[1,2]$. The intestinal tract is the largest immune organ in the human body. The intestinal epithelial barrier allows absorption of dietary nutrients and prevents passage of pathogens and toxins into the body. Severe insults have a negative impact on the intestinal environment, which may decrease intestinal barrier function and cause bacterial translocation. We previously reported that esophagectomy in patients with esophageal cancer disturbed the intestinal microbiota and decreased the concentrations of organic acids, while the administration of perioperative synbiotics significantly shortened the duration of the postoperative systemic inflammatory response syndrome and nonsignificantly reduced postoperative infectious complications by maintaining the intestinal microbiota and the concentrations of organic acids [3].

The fermentation of carbohydrates by the intestinal microbiota produces organic acids. Among them, short chain fatty acids (SCFAs) play a variety of important roles in maintaining intestinal mucosal barrier function and preventing bacterial translocation [4]. Bacterial translocation, which can cause infectious complications, is the passage of microbes or their products from the gastrointestinal tract across the mucosal barrier to extraintestinal sites, such as the mesenteric lymph nodes, liver, or bloodstream. Several investigators demonstrated that severe insults, such as major surgery and the severe systemic inflammatory response syndrome, disrupt the intestinal environment, in particular disturbing the intestinal microbiota and decreasing the concentrations of organic acids $[3,5,6]$. However, there is only one study concerning the association between the preoperative intestinal environment and postoperative infectious complications. Yokoyama et al. reported that low preoperative fecal concentrations of SCFAs were significantly associated with the occurrence of postoperative infectious complications in patients undergoing major hepatectomy with extrahepatic bile duct resection [7].

The aim of this study was to investigate the correlation between the preoperative fecal concentrations of organic acids and the occurrence of postoperative infectious complications in patients with esophageal cancer undergoing esophagectomy.

\section{Methods}

Patients

This study included 55 patients with thoracic esophageal cancer who underwent transthoracic esophagectomy at Osaka International Cancer Institute. These patients were included in our previous studies $[3,8]$. All patients received gastrointestinal fiberscopy and computed tomography scans of the neck, chest, and upper abdomen for tumor staging according to the TNM classification (7th edition) [9]. Patients underwent transthoracic subtotal esophagectomy with two- or three-field lymphadenectomy and reconstruction using the stomach or jejunum. An enteral feeding tube was inserted through the stomach or jejunum during the operation. Synbiotics were administered to all patients from 5 or more days before surgery to 21 days after surgery. The administered probiotics were Yakult BL Seichoyaku (Yakult Honsha, Tokyo), containing $1 \times 10^{8}$ live Bifidobacterium breve strain Yakult and $1 \times 10^{8}$ live Lactobacillus casei strain Shirota/g, and the prebiotics were galacto-oligosaccharides (Oligomate S-HP; Yakult Honsha). Before surgery, Yakult BL Seichoyaku (3 g/day) and galacto-oligosaccharides (15 g/day) were administered orally, and after surgery, Yakult BL Seichoyaku (2 g/day) and galacto-oligosaccharides (10 g/day) were administered through an enteral feeding tube or orally. Enteral feeding using a polymeric formula was initiated on postoperative day 1. Postoperative complications were assessed according to the Clavien-Dindo classification [10]. In this study, postoperative infectious complications were defined as those of grade II or higher.

\section{Fecal bacteriologic and organic acid analysis}

Fecal samples were collected 1 day before surgery. For measurement of the number of microbiota, fecal samples were placed into a tube containing RNAlater (Ambion, Austin, TX) and stored at $-20{ }^{\circ} \mathrm{C}$ until use. Total RNA was extracted from feces and the gut microbiota composition was examined using $16 \mathrm{~S}$ or $23 \mathrm{~S}$ ribosomal RNA-targeted reverse transcription-quantitative PCR using the Yakult Intestinal Flora-SCAN as described 
previously [11, 12]. For measurement of the concentrations of organic acids, fecal samples were placed into a vacant tube and stored at $-20^{\circ} \mathrm{C}$ until use. Feces were homogenized in perchloric acid and the concentrations of organic acids were measured using a Waters highperformance liquid chromatography system (Waters 432 Conductive Detector; Waters, Milford, MA) and a Shodex RSpak KC-811 column (Showa Denko, Tokyo, Japan) [13].

\section{Statistical analysis}

Statistical analyses were performed using JMP13 (SAS Institute) software. The relationships between postoperative infectious complications and clinical factors, numbers of fecal bacteria, or concentrations of fecal organic acids were evaluated by the chi-square test or the MannWhitney $U$ test. Multivariate analysis of infectious complications was performed using multiple logistic regression. Differences were significant at $p<0.05$.

The study protocol was approved by the Human Ethics Review Committees of Osaka International Cancer Institute and registered in the University Hospital Medical Information Network (http://www.umin.ac.jp; registration number ID UMIN000004704 and UMIN000006875). A signed consent form was obtained from each patient.

\section{Results}

\section{Patient characteristics and surgical outcome}

The clinical characteristics of the patients are presented in Table 1. The clinical disease stages were Stage I in 16 patients, Stage II in 15 patients, Stage III in 20 patients, and Stage IV (due to supraclavicular lymph node metastasis) in four patients. Neoadjuvant chemotherapy (NAC) or chemoradiotherapy was performed in 36 patients.

Postoperative infectious complications occurred in 10 patients $(18 \%)$, including pulmonary complications in

Table 1 Patient characteristics

\begin{tabular}{lll}
\hline Preoperative variables & & \\
Gender & male/female & $46 / 9$ \\
Age & median (range) & 64 (37-76) \\
Tumor location & upper/middle/lower & $6 / 30 / 19$ \\
Preoperative therapy & no/yes & $19 / 36$ \\
CT factor & T1/T2/T3/T4 & $12 / 15 / 26 / 2$ \\
CN factor & No/N1/N2/N3 & $25 / 19 / 10 / 1$ \\
CM factor & M0/M1 & $51 / 4$ \\
CStage & I/I/III/IV & $16 / 15 / 20 / 4$ \\
Intraoperative variables & & \\
Field of lymph node dissection & two-field/three-field & $15 / 40$ \\
Organ used in reconstruction & stomach/jejunum & $54 / 1$ \\
Operation time (min) & median (range) & 558 (411-755) \\
Blood loss (ml) & median (range) & 945 (370-3525) \\
\hline
\end{tabular}

seven, anastomotic leakage in one, pyothorax in one, and wound infection in two. There was no perioperative mortality.

Table 2 shows the association between preoperative and intraoperative factors and postoperative infectious complications. Postoperative infectious complications were more common in the elderly, but not significantly so $(p=0.073)$.

Table 3 shows the association between the number of representative fecal obligate anaerobes, administered probiotics, or fecal organic acid concentrations, and postoperative infectious complications. There was no significant difference in the number of representative fecal obligate anaerobes between patients with and without postoperative complications. The number of Bifidobacterium breve strain Yakult was nonsignificantly lower in patients with complications than in those without. By contrast, the concentrations of acetic acid and propionic acid were significantly lower in patients with complications ( $p=0.044$ and 0.032 , respectively). The concentration of butyric acid was nonsignificantly lower and the concentration of lactic acid was nonsignificantly higher in patients with complications. The concentration gap between acetic acid plus propionic acid plus butyric acid minus lactic acid (APB-L gap) was significantly lower in patients with complications than in those without. $(46.3 \pm 25.1 \mu \mathrm{mol} / \mathrm{g}$ of feces vs. $75.2 \pm 34.3 \mu \mathrm{mol} / \mathrm{g}$ of feces, respectively, $p=0.026$ ), as shown in Fig. 1. There was no significant correlation between fecal organic acid concentrations and the number of representative fecal obligate anaerobes (data not shown).

Table 4 shows the multivariate analysis for postoperative infectious complications. The APB-L gap cutoff value was determined using receiver operating characteristics curve analysis. Among factors with $p<0.2$ in the univariate analysis, the APB-L gap was the only factor significantly associated with postoperative infectious complications $(p=0.027)$.

\section{Discussion}

Patients with esophageal cancer who undergo esophagectomy have various risk factors that can negatively impact the intestinal environment, such as malnutrition due to cancer stenosis, preoperative therapy, surgical stress, use of antibiotics, postoperative fasting, and parenteral nutrition. If the preoperative intestinal environment is maintained, postoperative infectious complications may be reduced. This study revealed that a low APB-L gap was an independent risk factor for postoperative infectious complications. Preoperative concentrations of acetic acid, propionic acid, and butyric acid, which make up the majority of SCFAs (>95\%) [14], had a clinically important impact. These results are consistent with those of the previous 
Table 2 Correlation between preoperative or intraoperative factors and postoperative infectious complications

\begin{tabular}{|c|c|c|c|c|}
\hline & & Complication (-) & Complication (+) & $p$ value \\
\hline & & $(n=45)$ & $(n=10)$ & \\
\hline Gender & male/female & $37 / 8$ & $9 / 1$ & 0.55 \\
\hline Age & & $61.4 \pm 8.7$ & $66.8 \pm 4.9$ & 0.073 \\
\hline Tumor location & upper, middle / lower & $30 / 15$ & $6 / 4$ & 0.69 \\
\hline Preoperative therapy & no/yes & $17 / 28$ & $2 / 8$ & 0.28 \\
\hline cStage & $|-||/|||-\mid V$ & $19 / 26$ & $5 / 5$ & 0.65 \\
\hline BMI $\left(\mathrm{kg} / \mathrm{m}^{2}\right)$ & & $21.8 \pm 2.9$ & $21.0 \pm 1.6$ & 0.39 \\
\hline Operation time (min) & & $546 \pm 78$ & $589 \pm 78$ & 0.15 \\
\hline Blood loss (ml) & & $1045 \pm 517$ & $988 \pm 413$ & 0.98 \\
\hline
\end{tabular}

study by Yokoyama et al. [7]. In their study of patients undergoing major hepatectomy with extrahepatic bile duct resection, the preoperative fecal concentrations of acetic acid and butyric acid were significantly lower and the concentration of lactic acid was nonsignificantly lower in patients who developed postoperative infectious complications, and the acetic acid plus butyric acid minus lactic acid gap was an independent risk factor for postoperative infectious complications. Lactic acid is digested by obligate anaerobes to produce SCFAs [15]. Yokoyama et al. speculated that a low acetic acid plus butyric acid minus lactic acid gap may reflect an impaired intestinal environment and thus lead to postoperative infectious complications [7].

SCFAs play various roles in maintaining the intestinal environment, and contribute to the prevention of bacterial translocation. SCFAs stimulate the proliferation and differentiation of intestinal epithelial cells and the secretion of mucus by goblet cells $[16,17]$. They also maintain the acidity of the intestine and suppress the growth of harmful bacteria. Acetic acid, which is the most common organic acid, has an antimicrobial effect on harmful bacteria [18] and promotes the defensive functions of

Table 3 Correlation between fecal microbiota or organic acids and postoperative infectious complications

\begin{tabular}{|c|c|c|c|}
\hline & $\begin{array}{l}\text { Complication (-) } \\
(n=45)\end{array}$ & $\begin{array}{l}\text { Complication (+) } \\
(n=10)\end{array}$ & $\begin{array}{l}p \\
\text { value }\end{array}$ \\
\hline \multicolumn{4}{|l|}{ Fecal microbiota ( $\log _{10}$ cells/g of feces) } \\
\hline Clost. coccoides group & $9.46 \pm 0.56$ & $8.65 \pm 2.35$ & 0.77 \\
\hline Clost. leptam subgroup & $9.22 \pm 1.38$ & $8.51 \pm 2.36$ & 0.3 \\
\hline Bacteroides fragilis group & $9.29 \pm 1.70$ & $8.71 \pm 2.00$ & 0.52 \\
\hline Bifidobacterium & $9.80 \pm 1.12$ & $9.79 \pm 0.67$ & 0.35 \\
\hline Atopobium cluster & $9.21 \pm 0.74$ & $8.80 \pm 1.2$ & 0.23 \\
\hline Prevotella & $6.58 \pm 2.17$ & $5.94 \pm 2.38$ & 0.48 \\
\hline Total Lactobacillus & $7.56 \pm 0.16$ & $7.43 \pm 1.18$ & 0.50 \\
\hline Bifidobacterium breve strain Yakult & $8.03 \pm 1.23$ & $6.76 \pm 2.32$ & 0.11 \\
\hline Lactobacillus casei strain Shirota & $6.77 \pm 2.17$ & $5.93 \pm 2.38$ & 0.27 \\
\hline \multicolumn{4}{|l|}{ Fecal organic acid ( $\mu \mathrm{mol} / \mathrm{g}$ of feces) } \\
\hline Total organic acids & $80.9 \pm 29.8$ & $56.9 \pm 37.0$ & 0.11 \\
\hline Acetic acid & $56.0 \pm 25.8$ & $36.7 \pm 19.6$ & 0.044 \\
\hline Propionic acid & $16.0 \pm 10.2$ & $9.2 \pm 4.8$ & 0.032 \\
\hline Butyric acid & $4.5 \pm 3.9$ & $2.6 \pm 2.2$ & 0.19 \\
\hline Lactic acid & $1.3 \pm 2.6$ & $2.3 \pm 3.4$ & 0.12 \\
\hline Valeric acid & $0.88 \pm 0.59$ & $0.74 \pm 0.28$ & 0.39 \\
\hline Iso-valeric acid & $1.34 \pm 0.92$ & $0.90 \pm 0.32$ & 0.11 \\
\hline Formic acid & $0.97 \pm 1.5$ & $1.2 \pm 1.6$ & 0.57 \\
\hline Succinic acid & $2.3 \pm 6.0$ & $4.7 \pm 8.6$ & 0.67 \\
\hline
\end{tabular}




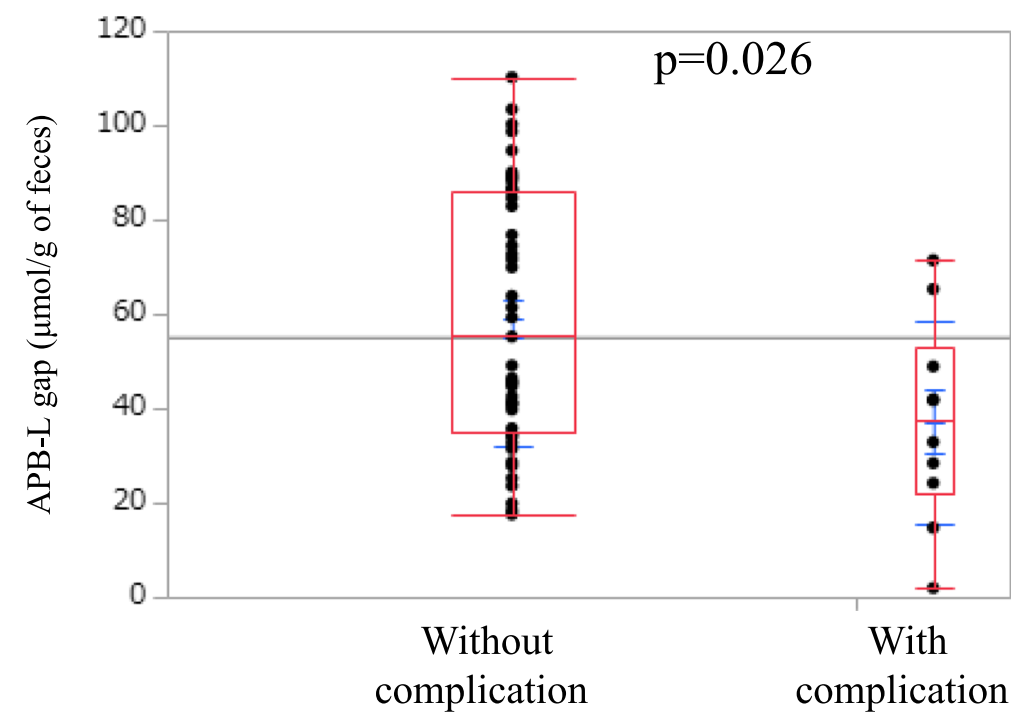

Fig. 1 APB-L gap in patients with and without postoperative infectious complications. The box shows the interquartile range. The top and bottom bars represent the maximum and minimum values, respectively

host epithelial cells [19]. Furthermore, Hsieh et al. reported that acetic acid strengthened tight junctions in Caco-2 monolayers [20]. Ohata et al. reported that propionic acid and butyric acid induced tight junction permeability in Caco-2 monolayer cells. Butyric acid is the major source of colonic epithelial cells, and induces the differentiation of regulatory $\mathrm{T}$ cells and ameliorates chronic intestinal inflammation in mice [21]. Thus, maintaining the concentration of SCFAs prior to iatrogenic gastrointestinal tract damage is useful for preventing bacterial translocation.

Organic acids are mainly produced in the large intestine by obligate anaerobes. In this study, there was no significant difference between the two groups in the number of representative fecal obligate anaerobes, as shown in Table 3. This result is inconsistent with that of the prior study mentioned above [7]. We previously reported that in patients with advanced esophageal cancer, NAC altered the intestinal microbiota and concentrations of organic acids [8]. In this study, about two-thirds of patients received preoperative chemotherapy or preoperative chemoradiotherapy. We performed a subanalysis in only patients who received preoperative therapy, examining the correlation between either the preoperative number of representative fecal obligate anaerobes or the fecal concentrations of organic acids and the incidence of postoperative infectious complications. There was no significant difference in the number of representative fecal obligate anaerobes between the two groups, while the concentrations of acetic acid and propionic acid were significantly lower in patients with complications than in those without ( $p=0.025$ and 0.022 , respectively, data not shown). The concentration of SCFAs is considered to reflect the function of the intestinal microbiota, which may be affected by preoperative therapy. Thus, the concentration of SCFAs but not the number of representative fecal obligate anaerobes was useful for predicting postoperative infectious complications in this study.

Acetic acid, which is the most common SCFA, is mainly generated by Bifidobacterium [19, 22]. In this study, the concentration of acetic acid was significantly lower in patients with complications than in those without. This may be because while the number of Bifidobacterium did not differ between the two groups, the number of Bifidobacterium breve strain Yakult, one of the administered probiotics, was nonsignificantly higher in patients without complications. Since Bifidobacterium breve strain Yakult can selectively metabolize galactooligosaccharide, it may increase the production of acetic acid [23]. In this study, although all patients received

Table 4 Multivariate analysis of postoperative infectious complications

\begin{tabular}{llll}
\hline & & Odds ratio $(95 \% \mathrm{Cl})$ & $p$ value \\
\hline Age & $\geq 70$ (vs. $\leq 69)$ & $1.24(0.28-5.80)$ & 0.77 \\
Operation time $(\mathrm{min})$ & $\geq 550$ (vs. $<550)$ & $3.47(0.79-18.8)$ & 0.11 \\
APB-L gap ${ }^{a}(\mu \mathrm{mol} / \mathrm{g}$ of feces) & $\leq 85$ (vs. $>85)$ & $8.04(1.24-160.34)$ & 0.027 \\
\hline
\end{tabular}

${ }^{a}$ : gap between acetic acid plus propionic acid plus butyric acid minus lactic acid 
synbiotics, including Bifidobacterium breve strain Yakult, for at least 5 days before surgery, the number of preoperative fecal Bifidobacterium breve strain Yakult was nonsignificantly lower in the patients with complication than in those without complication. It is considered that different patients had different responses to synbiotics. The mechanism of this variation is unclear. Currently, we administer perioperative synbiotics to all patients with esophageal cancer scheduled to undergo esophagectomy. Therefore, if a patient has low concentrations of SCFAs after receiving synbiotics, more careful attention should be paid during the perioperative period to prevent postoperative infectious complications. If high-performance liquid chromatography and reagents are available, the concentrations of fecal SCFAs can be measured in a few hours, and the cost is about 2000 Japanese yen per sample. Measurement of the concentrations of fecal SCFAs, which is a useful indicator for the occurrence of postoperative infectious complications, should be incorporated into daily clinical practice in the future.

Our study has several limitations. First, the number of enrolled patients was small. Second, about two-thirds of patients received preoperative chemotherapy or chemoradiotherapy, both of which alter the intestinal environment, while about one-third of patients received no preoperative therapy. Data of fecal microbiota and organic acid concentrations before and after NAC, that is, before surgery were available for 25 of the 55 cases enrolled in this study. Synbiotics were administered to these 25 patients during NAC. The number of bacteria administered as probiotics increased significantly after NAC, while no difference was found in the number of other bacteria before and after NAC. The concentration of butyric acid was significantly decreased after NAC $(p=0.0007)$. On the other hand, data of perioperative fecal microbiota and organic acid concentrations (before the administration of synbiotics, 1 day before surgery and 1 and 3 weeks after surgery) were available in 30 of the 55 patients enrolled in this study. Eleven patients received preoperative therapy without synbiotics and remaining 19 patients did not receive preoperative therapy. The number of Clostridium leptum subgroup before the administration of synbiotics was significantly larger in patients with preoperative therapy than in those without $(p=$ $0.047)$. No difference was observed in the number of Clostridium leptum subgroup at other time points, or in the number of other bacteria or organic acid concentrations between the two groups. In addition, the duration of synbiotics administration varied among individuals. This heterogeneity may have affected the results of our study. Third, there are no data concerning intestinal permeability, which correlates with the degree of bacterial translocation.

\section{Conclusion}

Preoperative fecal concentrations of acetic acid and propionic acid were significantly lower in patients who developed postoperative infectious complications than in those who did not. To reduce postoperative infectious complications after esophagectomy in patients with esophageal cancer, it may be useful to modulate the intestinal environment and maintain concentrations of fecal SCFAs.

\section{Abbreviations}

APB-L gap: The concentration gap between the acetic acid plus propionic acid plus butyric acid minus lactic acid; NAC: Neoadjuvant chemotherapy; SCFAs: Short chain fatty acids

\section{Acknowledgments}

We wish to express our sincere gratitude for the valuable assistance of Akira Takahashi, Satomi Atobe, Rie Date, and Takako Minami of the Yakult Central Institute in performing the fecal bacteriologic and organic acid analyses.

\section{Authors' contributions}

Study concepts: MM, KT. Study design: MM, KS. Data acquisition: MM, KT, TS, YM, TA, YK. Data analysis and interpretation: MM, HM, TA. Manuscript preparation: MM. Manuscript editing: KF, MY. Manuscript review: MY. All authors have read and approved the manuscript.

\section{Funding}

This study was partly supported by the Osaka Foundation for the Prevention of Cancer and Cardiovascular Diseases.

\section{Availability of data and materials}

The datasets used and/or analyzed during the current study are available from the corresponding author on reasonable request.

\section{Ethics approval and consent to participate}

The study protocol was approved by the Human Ethics Review Committees of Osaka International Cancer Institute and was registered in the University Hospital Medical Information Network (http://www.umin.ac.jp; registration number ID UMIN000004704 and UMIN000006875). A signed consent form was obtained from each patient.

Consent for publication

Not applicable.

\section{Competing interests}

The authors declare that they have no competing interests.

\section{Author details}

${ }^{1}$ Department of Surgery, Osaka International Cancer Institute, 3-1-69 Otemae, Osaka, Chuo-ku 541-8567, Japan. ${ }^{2}$ Department of Surgery, Osaka General Medical Center, 3-1-56 Bandai-higashi, Osaka 558-8558, Japan. ${ }^{3}$ Department of Gastroenterological Surgery, Graduate School of Medicine, Osaka University, 2-2 Yamadaoka Suita, Osaka 565-0875, Japan. ${ }^{4}$ Yakult Central Institute, 1796 Yaho, Kunitachi, Tokyo 186-8650, Japan.

Received: 17 September 2019 Accepted: 4 March 2020

Published online: 16 March 2020

\section{References}

1. Takeuchi $\mathrm{H}$, Miyata $\mathrm{H}$, Gotoh M, et al. A risk model for esophagectomy using data of 5354 patients included in a Japanese nationwide web-based database. Ann Surg. 2014;260:259-66.

2. Merkow RP, Bilimoria KY, McCarter MD, et al. Short-term outcomes after esophagectomy at 164 American College of Surgeons National Surgical 
Quality Improvement Program hospitals: effect of operative approach and hospital-level variation. Arch Surg. 2012;147:1009-16.

3. Tanaka K, Yano M, Motoori M, et al. Impact of perioperative administration of synbiotics in patients with esophageal cancer undergoing esophagectomy: a prospective randomized controlled trial. Surgery. 2012; 152:832-42.

4. Ohata A, Usami M, Miyoshi M. Short-chain fatty acids alter tight junction permeability in intestinal monolayer cells via lipoxygenase activation. Nutrition. 2005;21:838-47.

5. Kanazawa H, Nagino M, Kamiya S, et al. Synbiotics reduce postoperative infectious complications: a randomized controlled trial in biliary cancer patients undergoing hepatectomy. Arch Surg. 2005;390:104-13.

6. Shimizu K, Ogura H, Goto M, et al. Synbiotics decrease the incidence of septic complications in patients with severe SIRS: a preliminary report. Dig Dis Sci. 2009:54:1071-8.

7. Yokoyama Y, Mizuno T, Sugawara G, et al. Profile of preoperative fecal organic acids closely predicts the incidence of postoperative infectious complications after major hepatectomy with extrahepatic bile duct resection: importance of fecal acetic acid plus butyric acid minus lactic acid gap. Surgery. 2017;162:928-36.

8. Motoori M, Yano M, Miyata $\mathrm{H}$, et al. Randomized study of the effect of synbiotics during neoadjuvant chemotherapy on adverse events in esophageal cancer patients. Clin Nutr. 2017;36:93-9.

9. Sobin LH, Wittekind CH. TNM classification of malignant tumors. 7th ed. New York: Wiley-Liss; 2009.

10. Dindo D, Demartines N, Clavien PA. Classification of surgical complications: a new proposal with evaluation in a cohort of 6336 patients and results of a survey. Ann Surg. 2004;240:205-13.

11. Matsuda K, Tsuji H, Asahara T, et al. Establishment of an analytical system for the human fecal microbiota, based on reverse transcription quantitative PCR targeting of multicopy rRNA molecules. Appl Environ Microbiol. 2009; 75:1961-9.

12. Fujimoto J, Tanigawa $K$, Kudo $Y$, et al. Identification and quantification of viable Bifidobacterium breve strain Yakult in human faeces by using strain-specific primers and propidium monoazide. J Appl Microbiol. 2011;110:209-17.

13. Kamiya S, Nagino M, Kanazawa $H$, et al. The value of bile replacement during external biliary drainage: an analysis of intestinal permeability, integrity, and microbiota. Ann Surg. 2004;239:510-7.

14. Cook SI, Sellin JH. Review article: short chain fatty acids in health and disease. Aliment Pharmacol Ther. 1998;12:499-507.

15. den Besten $\mathrm{G}$, van Eunen $\mathrm{K}$, Groen AK, et al. The role of short-chain fatty acids in the interplay between diet, gut microbiota, and host energy metabolism. J Lipid Res. 2013;54:2325-40.

16. Guarner F, Malagelada JR. Gut flora in health and disease. Lancet. 2003;361: 512-9.

17. Willemsen LE, Koetsier MA, van Deventer SJ, van Tol EA. Short chain fatty acids stimulate epithelial mucin 2 expression through differential effects on prostaglandin $E_{1}$ and $E_{2}$ production by intestinal myofibroblasts. Gut. 2003; 52:1442-7.

18. Hayakawa M, Asahara T, Ishitani T, Okamura A, Nomoto K, Gando S. Synbiotic therapy reduces the pathological gram-negative rods caused by an increased acetic acid concentration in the gut. Dig Dis Sci. 2012;57:2642-9.

19. Fukuda $\mathrm{S}$, Toh $\mathrm{H}$, Hase $\mathrm{K}$, et al. Identification and quantification of viable Bifidobacterium breve strain Yakult in human faeces by using strain-specific primers and propidium monoazide. Nature. 2011;469:543-7.

20. Hsieh CY, Osaka T, Moriyama E, Date Y, Kikuchi J, Tsuneda S. Strengthening of the intestinal epithelial tight junction by Bifidobacterium bifidum. Physiol Rep. 2015;3:e12327.

21. Furusawa $Y$, Obata $Y$, Fukuda $\mathrm{S}$, et al. Commensal microbe-derived butyrate induces the differentiation of colonic regulatory T cells. Nature. 2013;504: 446-50.

22. Asahara T, Shimizu K, Nomoto K, Hamabata T, Ozawa A, Takeda Y. Probiotic bifidobacteria protect mice from lethal infection with Shiga toxin-producing Escherichia coli 0157:H7. Infect Immun. 2004;72:2240-7.

23. Shigehisa A, Sotoya H, Sato T, Hara T, Matsumoto H, Matsuki T. Characterization of a bifidobacterial system that utilizes galactooligosaccharides. Microbiology. 2015;61:1463-70

\section{Publisher's Note}

Springer Nature remains neutral with regard to jurisdictional claims in published maps and institutional affiliations.

\section{Ready to submit your research? Choose BMC and benefit from:}

- fast, convenient online submission

- thorough peer review by experienced researchers in your field

- rapid publication on acceptance

- support for research data, including large and complex data types

- gold Open Access which fosters wider collaboration and increased citations

- maximum visibility for your research: over $100 \mathrm{M}$ website views per year

At BMC, research is always in progress.

Learn more biomedcentral.com/submissions 